\title{
THE OUTBURST OF SYMBIOTIC NOVAE
}

\author{
H. NUSSBAUMER \\ Institute of Astronomy \\ ETH-Zentrum \\ CH-8092 Zürich \\ Switzerland
}

SUMMARY. We present the basic properties of symbiotic novae, and show how they differ from classical novae. Differences are observationally particularly noticeable in the luminosity curve, the rise of which is much slower, but its return to pre-outburst level takes much longer than in classical novae. Special emphasis is given to the intial phase, for which two different evolutionary paths have been observed. The outburst may lead into a supergiant phase lasting for several years, which is being followed by the nebular phase, or it may lead straight into the nebular phase. The total energy radiated during an outburst is at least as large as the energy loss of classical novae. Mass-loss during outburst may be sufficiently small to lead eventually to a supernova on the white dwarf.

\section{Introduction}

Symbiotic stars are double star systems; symbiotic novae are a subclass. They catch our attention when, during the course of a few months, they brighten by several magnitudes, usually $5 \lesssim \Delta m \lesssim 7$ and then remain at a steady high luminosity level for dozens of years. Up to now seven symbiotic systems can be confidently classed as symbiotic novae: AG Peg, RT Ser, RR Tel, V 1016 Cyg, HBV 475, HM Sge, and PU Vul. What is the cause of the outburst, and how does the outburst occur, where are the similarities and where the differences against classical novae? For an answer we shall look at four individual objects: HM Sge, AG Peg, RR Tel and PU Vul.

Symbiotic systems consist of a cool star, a hot star, and a nebula. The cool star is mostly an M giant, often a mira-type variable. The nebula is radiatively ionized; it is also detected at radio wavelength (e.g. Kwok et al. 1984, Seaquist and Taylor 1990). From the different ionization stages present, e.g. $\mathrm{N}^{+}$, $\mathrm{N}^{+2}, \mathrm{~N}^{+3}$, or from a Zanstra analyses we can deduce a radiation temperature. It turns out to be $50000<$ $T^{*}<200000$, or $T^{*} \approx 100000 \mathrm{~K}$ if you want just one typical value. - What is the origin of the nebula? Nussbaumer et al. (1988) did an abundance analyses for relative C/N/O abundances, and obtained the following results: The nebula shows practically the same relative $\mathrm{C} / \mathrm{N} / \mathrm{O}$ abundances as red giants, and they are definitely different from novae. They therefore conclude that the nebulae in symbiotic systems have their origin in the red giant.

To obtain luminosities and effective temperatures $T^{*}$, and thus their location in the HR-diagram, Mürset et al.(1991) have applied a modified Zanstra method to all symbiotic stars observed with IUE. It is based on He II $\lambda 1640$ and the underlying continuum. They find that the brightest among them are symbiotic novae, and that they lie not far below the Eddington limit. 


\section{The basic characteristics of symbiotic novae}

The following characteristics apply to all symbiotic novae: (1) The rise to maximum luminosity is much slower than in classical novae, it usually takes several months; (2) they remain at high luminosity for dozens of years, a typical decline time to pre-outburst luminosity is approximately 100 years. In their evolution to the nebular phase we distinguish two different paths. We demonstrate that point, together with some other fundamental features, with the help of four case stories: AG Peg, RR Tel, HM Sge, and PU Vul.

\subsection{HM Sagittae: $A$ rise in radiation temperature}

HM Sge began its outburst in 1975. The rise to maximum luminosity took about six months. The first spectra were taken from the ground late in 1976, approximately one year after the beginning of the outburst. They already show a nebular spectrum with $\mathrm{H}$ I, He I and [Ne III] among the lines seen. The flux ratio [Ne III] $\lambda 3868 /(\mathrm{H} \mathrm{I}+\mathrm{He} \mathrm{I} \lambda 3889)$ is very sensitive to variations in the radiation temperature, and the observations show that it increased from $T^{*} \approx 30000-40000 \mathrm{~K}$ in 1976 to $T \gtrsim 50000 \mathrm{~K}$ in June 1978 . Then high excitation emission lines appeared. The reversal in the flux ratio of N III $\lambda 1750 / N$ IV $\lambda 1485$ from $\approx 2$ before 1979 to $\approx 0.5$ after 1979 mirrors a further increase in the radiation temperature. The same information is extracted from the appearance of first [Ne IV], and later on [Ne V] and [Mg V], and from the reversal in the line flux ratio of $[\mathrm{Fe} \mathrm{VI]} \mathrm{and} \mathrm{[Fe} \mathrm{VII]} \mathrm{at} \lambda \lambda 5176,6087$. The radiation temperature reached $T^{*} \approx 100000 \mathrm{~K}$ in $1979, T^{*} \approx 125000 \mathrm{~K}$ in about 1985 , and $T^{*} \approx 170000 \mathrm{~K}$ in 1989 . Observations in 1990 indicate that $T^{*}$ is increasing further.

Although $T^{*}$ has increased by a factor 4 in the 14 years from 1976 to 1990, the luminosity has remained constant within a factor two. A description of these events is given in Nussbaumer and Vogel (1990). The outbursting star must have attained a radius of several solar radii at the end of its rise to maximum, its radiation temperature was then approximately $30000 \mathrm{~K}$, which is sufficient to create an appreciable lowly ionized nebula. Thus HM Sge went into the nebular stage practically immediately after reaching maximum luminosity. It then began to shrink and increase its radiation temperature. Since the beginning of the spectral observations the effective photospheric radius has been shrinking by more than a factor ten.

\subsection{AG Pegasi: A wind from the outbursting object.}

AG Peg is the oldest known symbiotic nova. Its outburst began between 1841 and 1855 . It has the longest documented history of all symbiotic stars. Observations at the turn from the $19^{\text {th }}$ to the $20^{\text {th }}$ century suggest that the outbursting nova had reached giant dimensions, but at that time there is no strong evidence for heavy mass-loss. The radiation temperature of the nova shell was probably below $30000 \mathrm{~K}$. In the 1920ies P-Cygni profiles appear and bear definite witness to heavy mass-loss from the nova. From the 1930ies onwards lines of N III, O III, N IV, and N V appear slowly in the course of thirty years, indicating a rise in $T^{*}$. During those years the luminosity appears to have remained approximately constant.

Whereas IUE observations of other symbiotic systems usually show line profiles which qualify that emission as nebular, AG Peg has at times shown lines of a hot wind, e.g. Nussbaumer (1990). They suggest final velocities of $v_{\infty} \approx 900 \mathrm{~km} / \mathrm{s}$. It is reasonable to interpret the profiles as being due to mass-loss from the hot star. IUE observations show a decline in line and continuum flux. Mürset et al.(1990) find $T^{*} \approx 100000 \mathrm{~K}$ for the full $1978-1989$ period. The luminosity has been steadily declining from $L=1400 L_{\odot}$ in 1978 to $L=460 L_{\odot}$ in 1989 . This corresponds to a shrinking in the radius of the hot component from $R=0.12 R_{\odot}$ in 1978 to $R=0.07 R_{\odot}$ in 1989 .

AG Peg shows (a) that the outburst of a symbiotic nova may last for over one hundred years, and (b) that the hot star in the symbiotic system may well have a wind of its own. 


\section{3. $R R$ Telescopii: The initial quasi-stable $F$ supergiant atmosphere.}

The hot component of $\mathrm{RR}$ Tel has now a radiation temperature of $T^{*} \approx 140000 \mathrm{~K}$. However, that was different between 1944 and 1955. In 1944 its outburst began. The system brightened from 13 to $7 \mathrm{mag}$. Thackeray (1977) describes the object: The luminosity showed a flat maximum from 1944 to 1949 , its spectrum was that of a F supergiant. Thackeray (1950) relates that in June 1949 it showed a pure absorption spectrum, type supergiant F, in August some of the former absorption lines had disappeared and by the middle of September it had turned into an emission spectrum where mainly H I and Fe II were present. Pottasch and Varsavsky (1960) analyse the change in the spectrum. Within one year the temperature rose from $7000 \mathrm{~K}$ to $12500 \mathrm{~K}$, the absorption lines disappeared and were replaced by $\mathrm{H} \mathrm{I}$ and Fe II emission. Between 1951 and 1952 a nebular spectrum evolved.

In RR Tel we see an early outburst equilibrium in a bloated state. Its effective photosphere resembles that of an $\mathrm{F}$ type supergiant. That configuration lasted for about six years. Then it changed: The radiation source grew hotter, a nebula appeared, and we slide into an evolution similar to that of HM Sge.

\subsection{PU Vulpeculae: From $F$ supergiant to the nebular phase.}

The outburst began in late 1977. Its rise by about 5 mag to maximum brightness $m_{\mathrm{B}} \approx 9$ took approximately one year. The system contains an M4 giant as cool component. The early spectra are of the supergiant middle F type. In 1987 a new phase began. Maitzen et al.(1987) report that PU Vul had entered a phase of increasing emission line strength. They mention in particular the singly ionized species Fe II, $\mathrm{Mg}$ II, Si II. This was the time when the spectrum changed into that of an A-supergiant, as seen in the IUE spectra from 1987 until late 1989 . This implies an increase in the radiation temperature from $\approx 6000 \mathrm{~K}$ to above $10000 \mathrm{~K}$. In 1990 the A-type spectrum has gone, and we see the emergence of a nebular spectrum. We find C III, N III, O III, Si III, and Fe III. But Fe II and O I begin to disappear. Looking at the spectra in more detail, we find that a lowering of the nebular densities from values probably above $10^{11} \mathrm{~cm}^{-3}$ to values below $10^{9} \mathrm{~cm}^{-3}$ must have occurred, and that the radiation temperature must have reached values of $30000 \mathrm{~K}$ to $40000 \mathrm{~K}$. But there is more! We see C IV and Si IV as broad wind lines with emission on the red, and absorption on the blue side, the corresponding velocities reach $500 \mathrm{~km} / \mathrm{s}$. Thus, the outbursting component is now shedding mass in form of a medium fast wind.

PU Vul gives us another important clue. In 1980 the hot outbursting star was eclipsed for a full year. During that time the former M4 giant spectrum was seen. This is fine evidence that the outburst does indeed occur on the hot companion, and that it hardly affects the M giant.

With its quasi-stable F-supergiant phase, that lasted for about nine years, PU Vul reminds us much of RR Tel. The transition to the nebular phase began in 1990 and we expect it to evolve as did RR Tel and HM Sge. In addition PU Vul proves the continued and practically undisturbed existence of the $M$ giant in the system. PU Vul also reminds us of AG Peg with its occasional showing up of wind lines with velocities of several hundred $\mathrm{km} / \mathrm{s}$. This wind is due to the hot star and we are particularly interested about relative $\mathrm{C} / \mathrm{N} / \mathrm{O}$ abundances of this early nebula which is most probably made up of material from the outbursting object and should therefore carry the traces of the underlying nuclear synthesis.

\section{Theoretical work on the outburst of symbiotic novae}

That symbiotic novae may be due to mass-accretion on a white dwarf and subsequent nuclear shell burning has been suggested some time ago. Corresponding fusion events have been studied, e.g. Paczyńsky and Zytkow (1978); Sion et al.(1979), Iben (1982), Kenyon and Truran (1983), Sion and Starrfield (1986). Livio 
et al.(1989), testing different parameter combinations, show that a slow nova type outburst is expected for a $1 M_{\odot}$ white dwarf with accretion rates of $\approx 10^{-8} M_{\odot} / \mathrm{yr}$. After a total amount of $\approx 10^{-5} M_{\odot}$ has been accumulated, an outburst of long duration occurs, that is, 20 years or longer. During outburst the envelope absorbs energy generated by nuclear burning at the foot of the envelope of the white dwarf, and it expands greatly. The expansion may lead to mass-ejection through an optically thick wind, e.g. Kato and Hachisu (1989), or it may simply lead to an extended shell, e.g. Shara et al.(1978). The mass of the shell decreases because of mass-loss and nuclear burning, or due to nuclear burning alone when mass-loss ceases. When nuclear burning ceases, the outbursting star returns to its pre-outburst state.

\section{The present picture of a symbiotic nova}

Combining theoretical work with observational data accumulated over the past 150 years, we can trace fairly clear contours of a symbiotic nova. The symbiotic nova happens after the originally heavier of the two stars has become a white dwarf, and accumulated the required skin of hydrogen-rich material by wind accretion from mass lost by the red giant. During outburst the expanding shell reaches luminosities in the neighbourhood of the Eddington luminosity. The rise to maximum luminosity takes many months. It may then remain for a few years in an equilibrium situation with an atmosphere of a type $F$ supergiant, or it may, after initial expansion, evolve straight into an object with a hot shrinking photosphere, where temperatures rise relatively fast to $100000 \mathrm{~K}$ and subsequently well above.

There is another important qualitative difference against classical novae. In the narrrow binary system of the classical nova a common envelope will develop during outburst, which will benefit from energy transfer extracted from the binary motion. This is likely to strongly influence the outburst. A symbiotic nova occurs in a relatively wide binary, and the outburst is an undisturbed thermonuclear event.

Classical novae are accompanied by heavy mass-loss. In symbiotic novae this is more ambiguous. The transition to the nebular phase may be accompanied by considerable mass-loss from the outbursting object. Heavy mass-loss may re-occur at later stages. Where such winds were observed, final velocities of $400-900$ $\mathrm{km} / \mathrm{s}$ were found. In the nebular stage the radiation temperature increases up to values of $T^{*} \approx 200000 \mathrm{~K}$. The increase in $T^{*}$ is accompanied by a shrinking of the effective photosphere of the expanded shell. The luminosity remains approximately constant during that period. The sizes of the hot component found by Mürset et al.(1991) lie typically between 0.01 and $0.5 R_{\odot}$. This is compatible with the assumption that symbiotic novae occur on a white dwarf, and that they return to a white dwarf at the end of the outburst. The total outburst lasts for many dozens of years, and 100 years may be a typical duration.

Although there is now sufficient evidence that outbursting symbiotic stars may suffer mass-loss, the available observations suggest, that the loss is considerably less than in novae. Thus, if the hot companion in a symbiotic system has initially a mass not much below the Chandrasekhar limit, accretion from the wind of the M giant may eventually provide sufficient mass for a supernova event on the hot star.

The total energy output in symbiotic novae is at least as high as in classical novae. For classical novae the total bolometric luminosity is $10^{45}-10^{46}$ erg (Gallagher and Starrfield, 1978). Mürset and Nussbaumer (1991) find for symbiotic novae $(4 \pm 3) \times 10^{46} \mathrm{erg}$. This amounts to an equivalent hydrogen mass of $(3 \pm 2) \times 10^{-6} M_{\odot}$.

Prior to outburst hydrogen-rich material has to be accreted from the mass lost by the cool component. For the cool component Mürset et al.(1991) find typical mass-losses of $\dot{M}=10^{-7} M_{\odot} /$ year, the uncertainty being about a factor 10 . This mass-loss has to provide the accretion rates of $\dot{M}=10^{-8} M_{\odot} /$ year required according to Livio et al.(1989). 
A final remark about interaction. Interaction is at least twofold. There is the pre-outburst mass-loss by the cool giant of which a fraction is collected by the accompanying white dwarf, leading to the slow nova outburst. There may be another kind of interaction after the onset of the outburst. This occurs in the nebula, away from the two stars. It happens when the outbursting component develops a wind on its own. We then find a region where two stellar winds collide. It is likely to be the site of X-ray emission which is occasionally observed in symbiotic systems, and it may also be at the origin of observed jet structures.

\section{References}

Gallagher, J.S., Starrfield, S.G.: 1978, Ann.Rev.Astron.Astrophys.16, 171

Iben, I.: 1982, Astrophys.J., 259, 244

Kato, M., Hachisu, I.: 1989, Astrophys.J., 346, 424

Kenyon, S.J., Truran, J.W.: 1983, Astrophys.J., 273, 280

Kwok, S., Bignell, R.C., Purton, C.R.: 1984, Astrophys.J. 279, 188

Livio, M., Prialnik, D., Regev, O.: 1989, Astrophys.J., 341, 299

Maitzen, H.M., Schnell, A., Hron, J.: 1987, IAU Circ.No. 4474

Mürset, U., Nussbaumer, H.: 1991, (this meeting)

Mürset, U., Nussbaumer, H., Schmid, H.M., Vogel, M.: 1991, Astron. Astrophys. (in press)

Nussbaumer, H.: 1990, Evolution in Astrophysics, ESA SP-310, p.87

Nussbaumer, H., Schild, H.R., Schmid, H.M., Vogel, M.: 1988, Astron. Astrophys. 198, 179

Nussbaumer, H., Vogel, M.: 1990, Astron. Astrophys. 236, 117

Paczyński, B., Żytkow, A.: 1978, Astrophys.J. 222, 604

Pottasch, S.R., Varsavsky, C.M.: 1960, Ann.Astrophys. 23, 516

Seaquist, E.R., Taylor, A.R.: 1990, Astrophys.J. 349, 313

Shara, M.M., Prialnik, D., Shaviv, G.: 1978, Astron.Astrophys.61, 363

Sion, E.M., Acierno, M.J., Tomzcyk, S.: 1979, Astrophys.J. 230, 832

Sion, E.M., Starrfield, S.G.: 1986, Astrophys.J. 303, 130

Thackeray, A.D.: 1950, Monthly Not.Roy.Astron.Soc. 110, 45

Thackeray, A.D.: 1977, Mem.Roy.Astron.Soc. 83, 1 